\title{
CEFALÉIA ASSOCIADA A INDICADORES DE TRANSTORNOS DE ANSIEDADE EM UMA AMOSTRA DE ESCOLARES DE RIBEIRÃO PRETO, SP
}

\author{
Maria Angela Marchini Gorayeb ${ }^{1}$, Ricardo Gorayeb ${ }^{2}$
}

\begin{abstract}
RESUMO - Avaliaram-se 374 crianças de 8 a 13 anos de idade em escolas públicas de Ribeirão Preto, objetivando averiguar a prevalência de queixas de cefaléia e sua associação com indicadores de transtornos de ansiedade. Os pais responderam um questionário sobre a presença e frequência de cefaléia e de comportamentos/sintomas associados a transtornos de ansiedade. As crianças foram avaliadas através da escala de ansiedade "O que Penso e Sinto". Das 374 crianças, 45,4\% nunca se queixaram de cefaléia; 41,2\%, ocasionalmente e 13,5\%, frequentemente. Entre aquelas que se queixam frequentemente de cefaléia, encontrou-se maior número de meninas, índice de ansiedade mais elevado e maior frequência de bruxismo e agitação. A alta prevalência de cefaléia na amostra e sua associação com maior frequência de bruxismo é compatível com dados epidemiológicos norteamericanos e brasileiros. Os resultados apontam para uma associação entre queixas frequentes de cefaléia e elevado índice de ansiedade.
\end{abstract}

PALAVRAS-CHAVE: cefaléia, ansiedade, infância, escolares.

\begin{abstract}
Association between headache and anxiety disorders indicators in a school sample from Ribeirão Preto, Brazil
ABSTRACT - A sample of 374 public elementary and junior high school students aged 8 to 13 years were evaluated to assess the prevalence of frequent headache complaints, associated with indications of anxiety disorder. Parents answered a questionnaire to investigate the presence and frequency of headache and of behaviors/symptoms that might indicate the presence of anxiety disorders. Children were evaluated using the Revised Children's Manifest Anxiety Scale adapted to this population. Among them, 45.4\% had never had a headache complaint; $41.2 \%$ had occasional headache complaints; and $13.5 \%$ had frequent headache complaints. Among children with frequent headache complaints there were more girls than boys, higher anxiety scores, higher occurrence of bruxism, and higher frequency of agitation. The high prevalence of headache and its association with bruxism in this sample is compatible with North American and Brazilian epidemiological data. Results show an association between frequent headache complaints and high anxiety scores.
\end{abstract}

KEY WORDS: headache, anxiety, childhood, schoolchildren.

As queixas de cefaléia são comuns na infância. Ao redor de dois a três anos de idade atingem 4,3\% das crianças, aumentando progressivamente até o início da vida escolar ${ }^{1}$. Dados epidemiológicos norte-americanos indicam que, ao redor dos 15 anos de idade, $15,6 \%$ das crianças apresentam cefaléia frequentemente e $54 \%$ têm cefaléia menos frequente, somando um total de $69,9 \%$ de prevalência de queixa de cefaléia ${ }^{2}$. Um levantamento das queixas de cefaléia apresentadas por adolescentes de 13 anos de idade na Finlândia concluiu que a queixa é apresentada por $81 \%$ dos jovens nesta faixa de idade 3 . Estudos sobre a cefaléia infantil no Brasil, embora ainda escassos, apontam para a alta frequência de ocorrência do quadro e destacam a gravidade das cefaléias como problema de saúde pública também em nosso país. O estudo realizado por Barea e col. ${ }^{4}$, em Porto Alegre RS, com 538 estudantes de 10 a 18 anos, encontrou a ocorrência de $72,3 \%$ de cefaléia do tipo tensional e de $9,9 \%$ de migrânea, no último ano, entre os indivíduos. Num outro estudo realizado em Santos SP, com 248 estudantes entre 7 a 15 anos de idade, um total de $43,1 \%$ das crianças entrevistadas receberam algum tipo de diagnóstico de cefaléia crônica diária primária (CCDP) pelos pesquisadores 5 .

\footnotetext{
Faculdade de Medicina de Ribeirão Preto, Universidade de São Paulo (FMRPUSP), Ribeirão Preto SP, Brasil: ${ }^{1}$ Mestre em Saúde Mental, 2Professor Associado. Agência financiadora: FAPESP processo n. ${ }^{\circ}$ 91/577-5.
} 
A prática clínica tem levado os profissionais que trabalham com pacientes com cefaléia a aventarem uma possível relação entre cefaléias e psicopatologia. Uma pesquisa pioneira na área foi realizada por Wolff em 1937 com pacientes adultos com diagnóstico de migrânea e levou-o a propor a existência de uma "personalidade migranosa: indivíduos tímidos, obedientes, inflexíveis e obstinados" ${ }^{\prime \prime}$. Tal teoria, embora atualmente abandonada pelos pesquisadores, chamou a atenção para a necessidade de investigação da correlação entre cefaléias e fatores psicológicos e, desde então, este assunto vem sendo extensamente pesquisado?.

Os dados mais consistentes resultam da investigação da comorbidade entre cefaléias, no caso CCPD, e ansiedade, demonstrando forte relação entre os quadros, tanto em amostras clínicas quanto populacionais ${ }^{8}$. A relação entre cefaléia e ansiedade pode aparecer tanto em casos onde os pacientes apresentam um diagnóstico de distúrbio de ansiedade, como Síndrome do Pânico ${ }^{9}$, ou também quando o paciente apresenta elevados índices de ansiedade como característica de personalidade, mas não apresenta nenhum distúrbio de comportamento. Tal fato levou pesquisadores a estabelecerem normas especiais da Escala de Ansiedade Traço-Estado de Spielberger para pacientes com migrânea e cefaléia do tipo tensional ${ }^{10}$. As escalas de ansiedade também são usadas para avaliar crianças com diversos quadros clínicos, incluindo cefaléias. Um importante instrumento utilizado em diversas culturas é o RCMAS (Revised Children's Manifest Anxiety Scale) ou What I Think and Feel. ${ }^{11,12}$. É preciso enfatizar que a identificação de doenças que ocorram concomitantemente com as CCDP é importante para o planejamento do tratamento. A identificação de populações com elevado risco para desenvolvimento da doença permite a adoção de estratégias preventivas ${ }^{13}$.

O objetivo deste trabalho foi identificar, em uma população de estudantes, a prevalência de queixas de cefaléia associada a comportamentos/sintomas indicativos de transtorno de ansiedade. Foi também objetivo testar a sensibilidade da escala de ansiedade infantil 'O Que Penso e Sinto' (adaptação da RCMAS para a população de Ribeirão Preto) ${ }^{14}$.

\section{MÉTODO}

Para atender aos objetivos propostos foi realizado um estudo transversal descritivo, utilizando-se de uma amostra probabilística, estratificada pela variável sócio-econômica e por cotas pelas variáveis sexo e idade.

A amostra de indivíduos continha 374 estudantes de oito a treze anos, sendo 190 do sexo feminino e 184 do
Tabela 1. Distribuição da amostra por sexo e idade.

\begin{tabular}{cccc}
\hline $\begin{array}{c}\text { Idade } \\
\text { anos }\end{array}$ & $\begin{array}{c}\text { Número } \\
\text { de Sujeitos }\end{array}$ & Masculino & Feminino \\
\hline 8 & 65 & 32 & 33 \\
9 & 63 & 30 & 33 \\
10 & 60 & 30 & 30 \\
11 & 59 & 28 & 31 \\
12 & 66 & 34 & 32 \\
13 & 61 & 30 & 31 \\
Total & 374 & 184 & 190 \\
\hline
\end{tabular}

sexo masculino, proporcionalmente distribuídos entre as séries de $2^{a}$ a $7^{a}$ do ensino fundamental. A Tabela 1 apresenta a distribuição dos indivíduos da amostra por sexo e idade. A abordagem aos indivíduos foi realizada através de uma amostragem de escolas, selecionadas com o auxílio da Delegacia de Ensino de Ribeirão Preto, de forma que a amostra contivesse, proporcionalmente, representantes das diferentes camadas sócio-econômicas de Ribeirão Preto. Foram selecionadas oito escolas públicas estaduais e municipais de ensino fundamental de Ribeirão Preto, S.P.

Utilizaram-se, para realização da pesquisa, os seguintes instrumentos:

- Escala de Ansiedade Infantil "O Que Penso e Sinto" $(\mathrm{OQPS})^{14}$. O instrumento utilizado constitui-se numa escala infantil para auto-aplicação, contendo 28 assertivas referentes a emoções e comportamentos associados à manifestação da ansiedade em crianças, às quais o indivíduo deve responder "sim" ou "não" ao fato de ocorrerem ou não a seu caso. A referida escala é originalmente Norte Americana (Revised Children's Manifest Anxiety Scale ou What I think and Feel $)^{15}$, e possui normas em diversos países. O estudo preliminar de normatização para a população infantil brasileira foi realizado concomitantemente com a coleta de dados aqui apresentados, que constituem-se no teste de sensibilidade do instrumento ${ }^{16}$.

O tipo de assertiva apresentada na escala é, por exemplo: "Eu acho difícil tomar decisões. ( ) Sim ( ) Não". O resultado é obtido pela somatória de respostas "sim", podendo então variar de 0 a 28 pontos, o que indicará a gradação da ansiedade.

- Questionário aos Pais (elaborado pelos pesquisadores, contendo três questões fechadas para investigar: 1 a presença de queixa de cefaléia pela criança; 2 - frequência de apresentação da queixa; 3 - presença de comportamentos/sintomas de ansiedade, a saber: enurese, sudorese, bruxismo, gagueira, agitação, onicofagia e tique). É importante citar que a escolha dos sete sintomas investigados foi baseada na experiência dos pesquisadores como psicólogos clínicos que selecionaram os itens mais facilmente observáveis por indivíduos leigos, como os pais, a partir do questionário $A_{2}$ de Rutter ${ }^{17}$. 
O Questionário aos Pais foi enviado para os pais dos alunos de todas as classes de segunda a oitava séries do ensino fundamental das oito escolas públicas selecionadas para o estudo, através dos próprios estudantes, juntamente com uma carta de consentimento informado. No prazo de uma semana a escala de ansiedade foi aplicada nos estudantes que haviam trazido o termo de consentimento assinado e o questionário respondido pelos pais. Todas as crianças foram testadas em grupos, em suas próprias salas de aula, pela pesquisadora que desconhecia até o momento o resultado dos questionários respondidos pelos pais (estudo cego), utilizando-se de instrução padronizada para a aplicação do instrumento ${ }^{14}$. Os indivíduos cujas escalas apresentavam muitas questões deixadas em branco foram excluídos. O procedimento foi aprovado pelo comitê de Ética em Pesquisa do HCFMRP-USP, dentro de um projeto de pesquisa com objetivos mais amplos.

A análise estatística constituiu-se da aplicação dos testes de Mann-Whitney e " $t$ " de Student $(p<0,05)$ para comparação dos resultados médios, obtidos na escala de ansiedade, dos grupos 'Sem Queixa de Cefaléia' e 'Com Queixa Frequente de Cefaléia'. Para a comparação dos resultados de frequência de apresentação de sintomas de ansiedade dos grupos 'Com Queixa Frequente de Cefaléia' e 'Sem Queixa de Cefaléia,' utilizou-se do teste de quiquadrado ou teste Exato de Fisher, em função do número de casos em cada categoria de sintoma.

\section{RESULTADOS}

Inicialmente houve um total de 472 indivíduos cujos pais assinaram o consentimento para que os filhos participassem do estudo e responderam ao questionário. No entanto 98 indivíduos foram excluídos devido à forma como responderam à escala de ansiedade (grande número de questões em branco ou com respostas inválidas), sendo portanto 374 o número total da amostra utilizada.

Todos os indivíduos cujos questionários continham resposta "Não" à questão número 1 (um), foram considerados indivíduos que não apresentam queixa de cefaléia ou seja, 'Grupo Sem Queixa de Cefaléia'.

Aqueles cujos questionários continham resposta "Sim" à questão número 1 (um) e resposta "todos os dias" ou "várias vezes por semana" à questão número 2 (dois), foram identificados como indivíduos que apresentam 'Queixa Frequente de Cefaléia.' Os demais indivíduos $(n=153)$, cujos pais responderam "Sim" à questão número 1 (um) do Questionário de Pais, afirmando a presença de queixa de cefaléia pela criança, mas relataram frequência menor que uma vez por semana, foram considerados como indivíduos que queixam de cefaléia ocasionalmente e não considerados para as comparações estatísticas apresentadas.

As análises comparativas apresentadas a seguir foram feitas utilizando-se 221 indivíduos da amostra.
Tabela 2. Distribuição dos indivíduos do grupo "com queixa freqüente de cefaléia", por sexo e idade.

\begin{tabular}{ccc}
\hline Idade & Sexo Masculino & Sexo Feminino \\
\hline 8 & 4 & 3 \\
9 & 2 & 6 \\
10 & 4 & 7 \\
11 & 0 & 4 \\
12 & 2 & 7 \\
13 & 5 & 6 \\
Total & $17 *$ & $33^{*}$ \\
\hline * A diferença entre tais valores é significativa, com $p=0,007$, pelo teste do \\
qui-quadrado.
\end{tabular}

Considerou-se relevante a comparação entre as crianças do grupo 'Sem Queixa de Cefaléia' e os indivíduos do grupo 'Com Queixa Frequente de Cefaléia'.

A Tabela 2 mostra a distribuição dos indivíduos 'Com Queixa Frequente de Cefaléia', por sexo e idade.

A amostra de indivíduos 'Sem Queixa de Cefaléia' é composta de 74 meninas e 97 meninos e a amostra de indivíduos com 'Queixa Frequente de Cefaléia' é composta de 33 meninas e 17 meninos (Tabela 2). $\mathrm{Na}$ amostra de indivíduos que apresentam queixa frequente de cefaléia foi encontrado um número maior de crianças do sexo feminino ( $p=0,007$, teste qui-quadrado).

Os resultados da Escala de Ansiedade OQPS e os dados obtidos na questão número 3 (três) do Questionário de Pais, que investiga a presença de comportamentos/sintomas de ansiedade, foram comparados entre os dois grupos.

Observa-se então que da amostra de 374 crianças, $45,4 \%$ nunca queixam de cefaléia (171 indivíduos "Sem Queixa de Cefaléia") e 13,4\% (50 indivíduos "Com Queixa de Cefaléia") apresentam queixa frequente de cefaléia. Os 41,2\% restantes (153 indivíduos) queixam-se ocasionalmente, somando portanto um total de 54,6\% com alguma queixa de cefaléia na amostra total de 374 crianças estudadas.

Observa-se pela Tabela 3 que, dentre os sete comportamentos/sintomas investigados através do Questionário aos Pais (enurese, bruxismo, agitação, onicofagia, sudorese, gagueira e tiques), somente bruxismo e agitação ocorreram em maior frequência entre as crianças do grupo 'Com Queixa Frequente de Cefaléia' ( $p=0,06$ e $p=0,008$ respectivamente, teste do qui-quadrado). Nesta tabela os dados são apresentados em porcentagem para permitir melhor comparação entre os grupos. O teste Exato de Fisher foi utilizado quando era mais apropriado, em função do número de sujeito em cada categoria de sintoma. 
Tabela 3. Distribuição de sintomas de ansiedade apresentados pelos indivíduos dos grupos "com queixa freqüente de cefaléia" e 'sem queixa de cefaléia".

\begin{tabular}{lccc}
\hline $\begin{array}{l}\text { Sintomas de } \\
\text { ansiedade }\end{array}$ & $\begin{array}{c}\text { Grupo com } \\
\text { queixa de } \\
\text { cefaléia (\%) }\end{array}$ & $\begin{array}{c}\text { Grupo sem } \\
\text { queixa de } \\
\text { cefaléia }(\%)\end{array}$ & $\begin{array}{c}\text { Níveis de } \\
\text { significância } \\
(p)\end{array}$ \\
\hline Agitação* & 56,0 & $33,92^{*}$ & $0,005^{*}$ \\
Onicofagia & 50,0 & 41,52 & 0,29 \\
Bruxismo* & 40,0 & $25,15^{*}$ & $0,04^{*}$ \\
Sudorese & 22,0 & 15,20 & 0,28 \\
Gagueira & 14,0 & 7,60 & 0,17 \\
Enurese & 8,0 & 6,43 & $0,75^{* *}$ \\
Tiques & 8,0 & 7,02 & $0,76^{* *}$ \\
\hline
\end{tabular}

*Diferenças significativas (teste do qui-quadrado), **teste exato de Fisher (demais cálculos feitos pelo teste do qui-quadrado).

A média de ansiedade obtida através da escala OQPS para o 'Grupo Sem Queixa de Cefaléia' é 11,24 pontos (mediana $=11,0$ ) e para o 'Grupo Com Queixa Frequente de Cefaléia' é 15,02 pontos (mediana = $14,5)$. Deve-se citar que, segundo dados normativos da escala "O Que Penso e Sinto", o resultado médio padrão é de 12,36 pontos com desvio padrão de 5,77 pontos. Apesar da variação dos resultados destes grupos estar incluída no intervalo de desvio padrão, uma análise de tais resultados nos testes de Mann-Whitney $\mathrm{e}$ " $\mathrm{t}$ " de Student demonstra uma diferença estatisticamente significativa entre os escores de ansiedade obtidos pelos grupos $(p=0.0005)$.

\section{DISCUSSÃO}

Uma revisão da literatura internacional mostra que a prevalência de cefaléia na infância varia muito em cada estudo apresentado. Vários fatores colaboram com este fato, desde a possibilidade de diagnósticos combinados até a dificuldade de investigação junto ao paciente infantil. Mas principalmente, como aponta o estudo de Arruda e col. ${ }^{18}$, é a existência de diferentes critérios utilizados para o diagnóstico das cefaléias que ocasiona divergências nos resultados dos levantamentos epidemiológicos. Em termos gerais, a prevalência de cefaléia na população infantil parece variar entre $54 \%$ a $89 \%$, se consideradas as cefaléias menos frequentes ${ }^{1-5,13}$. Pode-se dizer que os dados encontrados na amostra do presente estudo não são discrepantes de dados encontrados na literatura, levando a crer que a amostra de indivíduos é representativa da população e que os individuos do grupo 'Com Queixa Frequente de
Cefaléia', se avaliados clinicamente, poderiam vir a receber algum diagnóstico de CCDP ${ }^{19}$.

No presente estudo encontrou-se maior prevalência de queixas de cefaléia entre crianças do sexo feminino, fato que já foi observado em outros estudos, porém sofrendo variações conforme a faixa etária. Em um estudo realizado na Finlândia encontrou-se maior prevalência de cefaléia em indivíduos do sexo masculino, para os grupos com menos de oito anos de idade, e maior prevalência de cefaléia em indivíduos do sexo feminino, para os grupos com idades acima de oito anos $^{20}$. No estudo de Barea e col. ${ }^{4}$, em Porto Alegre RS, a frequência de cefaléia do tipo tensional foi maior para o sexo feminino, embora não tenha havido diferença quanto a sexo para aqueles cujos diagnósticos eram de migrânea. Neste estudo, o baixo número de indivíduos no grupo ‘Com Queixa Frequente de Cefaléia' ( 50 casos) não permitiu análise por faixa etária, e o modelo de estudo não possibilitava ou pretendia uma classificação diagnóstica.

Dentre os sete comportamentos/sintomas de ansiedade investigados pelo Questionário aos Pais (enurese; bruxismo; agitação; onicofagia; sudorese; gagueira e tiques) apenas dois, bruxismo e agitação, mostraram-se mais frequentes entre os indivíduos do grupo 'Com Queixa Frequente de Cefaléia'.

A maior ocorrência de bruxismo nesta população era esperada, pois vários estudos já mostraram a alta prevalência de bruxismo em pacientes com cefaléia. Por exemplo, no estudo de Aromaa et. al. ${ }^{21}$ uma investigação compreensiva da saúde de 106 indivíduos com queixa de cefaléia e 106 controles indicou que bruxismo e, consequentemente, alterações da junta têmporo-mandibular eram mais frequentes entre os indivíduos do grupo com queixa de cefaléia. Num estudo realizado em Ribeirão Preto SP, com 51 pacientes infantis com o diagnóstico de migrânea, comparados com 51 controles, o bruxismo foi identificado, juntamente com outros sete transtornos, como sendo um transtorno associado à migrânea infantil, além de um fator de risco que deve ser considerado no momento de realizar o diagnóstico diferencial da criança com queixa de cefaléia ${ }^{22}$.

Os resultados aqui apresentados permitem aos autores afirmarem que, as crianças 'Com Queixa Frequente de Cefaléia' apresentam uma elevação nos escores de ansiedade pela OQPS quando comparadas às crianças 'Sem Queixa de Cefaléia', o que atesta a sensibilidade psicométrica do instrumento ${ }^{16} \mathrm{em}$ sua versão adaptada para o português.

Resultados semelhantes de comorbidade entre 
cefaléias e ansiedade podem ser encontrados na literatura, como por exemplo no estudo realizado por Guidetti et. al. ${ }^{8}$ onde 330 pacientes infantis de uma clínica de cefaléia foram seguidos por oito anos e frequentemente avaliados por uma bateria de testes psicológicos. Este estudo mostrou uma associação significativa entre migrânea e transtornos de ansiedade, o mesmo não ocorrendo entre aqueles pacientes que apresentaram remissão dos sintomas de cefaléia.

O estudo de Merikangas ${ }^{23}$ da comorbidade entre CCDP e transtornos psicopatológicos indica a seguinte sequência de instalação de quadros: primeiramente um transtorno de ansiedade, seguida da cefaléia crônica e finalmente um quadro depressivo. Egger et al ${ }^{7}$, discordam desta relação linear mas confirmam a associação entre cefaléias, ansiedade e depressão. Independente do tipo de relação existente entre os quadros (linear ou não) o tratamento da ocorrência do primeiro quadro instalado, em determinado paciente ou população, poderia prevenir a evolução para algum dos outros quadros associados, diminuindo as consequências debilitantes sofridas pelos indivíduos atingidos.

O presente estudo aponta para a importância de um aprofundamento do estudo de comorbidade entre cefaléias e transtornos de ansiedade na população infantil brasileira, além da necessidade de um atendimento integrado médico-psicológico do paciente infantil com queixa de cefaléia, a fim de solucionar os diferentes aspectos envolvidos na problemática e garantir o melhor prognóstico para cada caso.

\section{REFERÊNCIAS}

1. Sillampää MD. Headache in children. In Olensen J (Ed) Headache classification and epidemiology. New York: Raven Press, 1994:273-281.

2. Rothner AD. Headaches in children and adolescents: classification and recommendation. Postgrad Med 1987;81:223-230.

3. Silampäa M. Prevalence of headache in prepuberty. Headache 1983;23:10-14.
4. Barea LM, Tamnhauser M, Rotta NT. An epidemiologic study of headache among children and adolescents of southern Brazil. Cephalalgia 1996;16:545-549.

5. Fragoso YD, Padeiro RP, Moura RT, Moriyama MG, Ribeiro RP. Cefaléia crônica na infância: estudo em uma população de estudantes em Santos, S.P. Migrâneas e Cefaléias 2000;3:23-29.

6. Wolff HG. Personality features and reactions of subjects with migraine. Arch Neurol Psychiatry 1937;37:895.

7. Egger HL, Angold A, Costello, J. Headaches and psychopathology in children and adolescents. J Am Acad Child Adolesc Psychiatry 1998;37: 951-958.

8. Guidetti V, Galli F, Fabrizi P, et al . Headache and psychiatry comorbidity: clinical aspects and outcome in an 8-year follow-up study. Cephalalgia 1998;18:455-462.

9. Stewart W, Breslau N, Keck PE Jr. Comorbidity of migraine and panic disorder. Neurology 1994;44(Supl.-7):23-27.

10. Blanchard EB, Andrasik F. Psychological testing and headache. In Blanchard EB, Andrasik F. Management of chronic headaches: a psychological approach. New York: Pergamon Press, 1985;23-33.

11. Labblé EE. Childhood muscle contraction headache: current issues in assessment and treatment. Headache 1988:30;430-434.

12. Larsson B, Mellin L, Dorbel A. Recurrent tension headache in adolescents treated with self help relaxation training and muscle relaxant drug. Headache 1990;30:665-671.

13. Silberstein SD, Lipton RB, Goadsby PJ. Epidemiology and impact of headache disorders. In Headache in clinical practice. Oxford: Isis Medical Media, 1998:19-30.

14. Gorayeb MAM. Adaptação, normatização e avaliação das qualidades psicométricas da RCMAS (Revised Children's Manifest Anxiety Scale) para uma amostra de escolares de oito a 13 anos de idade em Ribeirão Preto, SP. Dissertação de Mestrado, Faculdade de Medicina de Ribeirão Preto, Universidade de São Paulo. Ribeirão Preto, 1997.

15. Reynolds CR, Richmond BO. What I think and feel: a revised measure of children's manifest anxiety. Child Psychol 1978;6:271-280.

16. van Kolck OL. Técnicas de exame psicológico e suas aplicações no Brasil: testes de personalidade: 2.Ed Rio de Janeiro: Vozes, 1981.

17. Graminha SSV. A escala comportamental infantil de Rutter A2: estudos de adaptação e fidedignidade. Estudos de Psicologia 1994;11:34-42.

18. Arruda MA, Speciali JG, Ciciarelli MC, Bordini CA. Childhood migraine: diagnostic problems. Cephalalgia 1995;15(Suppl.16):82.

19. Raffaelli E, Silva WF, Martins OJ (tradutores) Classificação e critérios diagnósticos das cefaléias, nevralgias cranianas e dor facial. São Paulo: Lemos Editorial, 1997.

20. Sillanpää M. Changes in the prevalence of migraine and other headaches during the first seven school years. Headache 1983;23:15-19.

21. Aromaa M, Sillanpää ML, Rautava $P$, Helenius H. Childhood headache at school entry - a controlled clinical study. Neurology 1998;50:1729-1736.

22. Arruda MA, Speciali JG, Ciciarelli MC, Bordini CA. Risk factors and associated disorders of childhood migraine. Cephalalgia 1995; 15 (Suppl.16): 83.

23. Merikangas KR, Angst J, Isler H. Migraine and psychopathology: results of the Zurich cohort study of young adults. Arch Gen Psychiatry 1990;47:849-853. 